\title{
Isolated cerebellar vermis hypoplasia
}

INSERM

\section{Source}

INSERM. (1999). Orphanet: an online rare disease and orphan drug data base. Isolated cerebellar vermis hypoplasia. ORPHA:199630

Isolated cerebellar vermis hypoplasia is a rare, non-syndromic cerebellar malformation characterized by an underdeveloped cerebellar vermis. Patients may present a variable phenotype rang ing from normal neurodevelopment to motor and/or language delay, variable degrees of cognitive impairment, hypotonia, equilibrium disturbances, static/dynamic ataxia, oculomotor abnormalities, epilepsy and/or clumsiness. Behavioral disorders such as attention deficit hyperactivity disorder and generalized anxiety have also been reported. Brain MRI may reveal diffuse or selective (mostly posterior) vermian cerebellar hypoplasia and EEG may show focal paroxysms. 